\title{
Relaciones entre los bovinos criollos panameños y algunas razas criollas de Latinoamérica
}

\author{
Axel Villalobos-Cortés ${ }^{(1)}$, Amparo Martínez $^{(2)}$, José Luis Vega-Pla(3), Vincenzo Landi(2), Jorge Quiroz ${ }^{(4)}$, \\ Rubén Martínez ${ }^{(5)}$, Roberto Martínez López ${ }^{(6)}$, Phil Sponenberg(7), Eileen Armstrong ${ }^{(8)}$, \\ Delsito Zambrano( ${ }^{(9)}$, Jose Ribamar Marques ${ }^{(10)}$ y Juan Vicente Delgado(2)
}

\begin{abstract}
(1)Instituto de Investigación Agropecuaria, Laboratorio de Agrobiotecnología, Apartado 6-4391 El Dorado, Panamá. E-mail: villalobos. axel@gmail.com (2)Universidad de Córdoba, Departamento de Genética, Edificio Gregor Mendel, Campus de Rabanales, 14071 Córdoba, España. E-mail: ib2mamaa@uco.es ${ }^{(3)}$ Cría Caballar de las Fuerzas Armadas, Laboratorio de Investigación Aplicada, Carretera Madrid-Cádiz Km 397, 14071 Córdoba, España: E-mail: jvegpla@oc.mde.es (4)Instituto Nacional de Investigaciones Forestales, Agrícolas y Pecuarias, Km 1, Carretera Huimanguillo-Cárdenas, Huimanguillo, CP 86400, Tabasco, México. E-mail: quiroz.jorge@inifap.gob.mx (5) Universidad Nacional Lomas de Zamora, Departamento de Genética Animal, Ruta 4, Km 2, 1836 Llavallol, Argentina. E-mail: martinezruda@yahoo.com.ar (6)Universidad Nacional de Asunción (UNA), Campus de la UNA, San Lorenzo, Paraguay. E-mail: agrotulo@yahoo.com (7)Virginia Tech, Virginia-Maryland Regional College of Veterinary Medicine, Duck Pond Drive (0442), Blacksburg, Virginia 24061, USA. E-mail: dpsponen@vt.edu (8)Universidad de la República, Departamento de Genética y Mejora Animal, Chaná 2020, 11200 Montevideo, Uruguay. E-mail: eileen.armstrong@gmail.com (9)Universidad Técnica Estatal de Quevedo, Km 7, Vía El Empalme (entrada a Mocache), Finca Experimental La María, Ecuador. E-mail: delsitoz@yahoo.com ${ }^{(10)}$ Embrapa Amazônia Oriental, Caixa Postal 48, CEP 66095-100 Belém, PA, Brasil. E-mail: marques@cpatu.embrapa.br
\end{abstract}

Resumen - El objetivo de este trabajo fue establecer la relación genética entre poblaciones bovinas panameñas Guabalá y Guaymí y algunas poblaciones criollas de Latinoamérica. Se practicó un análisis factorial de correspondencias, análisis de varianza molecular, distancias genéticas, número medio de migrantes por población y los estadísticos $\mathrm{F}$ de Wright. Se evaluó la estructura de la población mediante un modelo Bayesiano, suponiéndose un número desconocido de K grupos diferentes genéticamente. El análisis factorial de correspondencias mostró que las poblaciones Guabalá y Guaymí se agrupan con los bovinos criollos mexicanos y el Texas Longhorn. Igualmente se observó menor diferenciación genética de las criollas panameñas con mexicanos y el Texas Longhorn. Los análisis de distancia genética también mostraron dados similares a los obtenidos por el Amova y por el análisis factorial de correspondencia, y se observó menor distancia entre poblaciones del norte y las panameñas, en comparación con las poblaciones del sur. La agrupación bayesiana permitió la asignación de los individuos a su respectivo grupo, con base en su semejanza genética, y proporcionó información acerca del número de poblaciones bajo el cual se originan. Hay una estrecha relación histórica, genética y geográfica de las poblaciones panameñas, criollas mexicanas y Texas Longhorn, a partir de las migraciones de sus precursores desde las Antillas hacia Panamá y México.

Términos de indexación: conservación, criollo, estructura genética, Guabalá, Guaymí, QTL.

\section{Relationships between Panamanians and some creole cattle landraces in Latin America}

Abstract - The objective of this work was to establish the genetic relationship between Guabalá and Guaymi cattle populations and some native ones of Latin America. Factorial correspondence analysis, analysis of molecular variance, genetic distances, average number of migrants per population and Wright's $\mathrm{F}$ statistics were performed. Population structure was assessed by a Bayesian model, assuming an unknown number of K genetically distinct groups. The correspondence analysis showed that the populations of Guabalá and Guaymí cluster with Mexican creole cattle and Texas Longhorn. Lower genetic differentiation of Panamanian creole with Mexican and Texas Longhorn was also observed. The analyses of genetic distances have also shown similar results to those obtained by Amova and by the factorial correspondence analysis, and the less distance was observed between north populations and Panamanian ones, in comparison with southern populations. Bayesian clustering permitted the assignment of individuals to their respective groups, based on their genetic similarity, and provided information on the number of cluster from which they originate. There is a close historical, genetic, and geographic relationship of Panamanian, Mexican, and Texas Longhorn populations due to the migration of precursors from the Caribbean islands to Panama and Mexico.

Index terms: conservation, local breeds, genetic structure, Guabalá, Guaymi, QTL.

Pesq. agropec. bras., Brasília, v.47, n.11, p.1637-1646, nov. 2012 


\section{Introducción}

En la historia de la conquista española, el año 1521 marca el inicio de la llegada de bovinos hacia tierra firme, y un proceso de evolución que generaría una gran diversidad de razas adaptadas al nuevo medio geográfico, que en lo sucesivo se les denominarían como razas criollas (Archivo General de Indias, 1521; Primo, 1992; Rodero et al., 1992; Villalobos Cortés et al., 2009a). Debido a sus características geográficas, Panamá se convirtió en un punto importante de dispersión de animales hacia el norte, por toda Centroamérica, y hacia el sur, durante la conquista del imperio Inca, actual Perú (Cortés Ramos, 2002).

Posterior al ingreso de las poblaciones bovinas a tierra firme, Panamá se estableció como puente de migración de bovinos hacia la región mesoamericana o norte de Panamá. Datos recabados sobre la vida de Pedrarias Dávila, Gobernador de Castilla del Oro y posteriormente Gobernador de Nicaragua, sugieren que fue responsable y el gestor de esta migración a lo largo del territorio panameño y en el resto de Centroamérica (Cortés Ramos, 2002; Guilhermo, 2003).

Por otro lado, la ganadería en el Perú fue desarrollada con base en los animales llevados por los primeros colonizadores. Estos animales hacían su primera escala en Panamá, para luego continuar hacia el Perú y el Ecuador. De esta forma, Panamá también se convirtió en el centro de acopio y distribución de bovinos para las colonias suramericanas (Villalobos Cortés et al., 2009a).

Las razas bovinas criollas Guaymí y Guabalá han sido identificadas y caracterizadas mediante marcadores microsatélites; estas poblaciones mostraron alta diversidad genética y valores de endogamia de bajos (Guaymí) a moderados (Guabalá). Además, mostraron un valor de diferenciación genética entre ambas razas cercano al 7\%, similares a los encontrados entre las poblaciones europeas (Villalobos Cortés et al., 2010). Bajo ese aspecto, las poblaciones Guaymí y Guabalá también fueron comparadas con razas cebuinas y taurinas, comúnmente encontradas en Panamá, y de las cuales podría presumirse que fueran una mezcla aleatoria. Sin embargo, los valores de diferenciación $\left(\mathrm{F}_{\mathrm{ST}}\right)$ calculados para las razas cebuinas (Gyr, Brahman, Sindi, Guzerat and Nelore) y taurinas (Holstein, Pardo Suiza y Hereford) fueron 0,21 y 0,10 , respectivamente, considerados altos (Villalobos Cortés et al., 2010).
El ganado Guaymí presenta diversidad de colores en la capa externa de la piel, su tamaño medio a la cruz es de 1,20 m de altura y 1,79 $\mathrm{m}$ de longitud corporal. Al nacer, los machos y las hembras pueden pesar $27 \mathrm{y}$ $28 \mathrm{~kg}$, respectivamente, con peso adulto promedio de $465 \mathrm{~kg}$. Este ganado se encuentra localizado en áreas de difícil acceso en la comarca Gnäbe-Bugle, región occidental de la República de Panamá, en la que se presupone ha sufrido un aislamiento geográfico, por lo menos en gran parte de los años posteriores a la llegada de los conquistadores.

Las características de la raza Guabalá son: capa externa de piel en colores rojo, berrendo y rubio. Tiene los cuartos traseros fuertes y bien formados, con cabeza grande y alargada, orejas pequeñas y cuernos largos y puntiagudos (Villalobos Cortés, 2010). Se les observa un pelo muy corto y brillante, posiblemente atribuida a un gen dominante llamado "slick", muy común en las razas localmente adaptadas, que les confiere propiedades aptas para el clima tropical (Olson et al., 2003).

Además de los métodos que se utilizan para determinar distancia y estructura genética de poblaciones, como árboles de distancia, análisis multivariante y análisis de varianza molecular, Pritchard et al. (2000) introdujeron un método para identificar poblaciones diferentes, considerándose que los individuos sean puros y provenientes de alguna de las $\mathrm{K}$ poblaciones, o bien bajo el supuesto que haya existido cruzamientos de sus ancestros; es decir, que una fracción $\mathrm{q}_{\mathrm{k}}$ del genoma de un individuo provenga de una subpoblación, dando la información de los ancestros (Falush et al., 2003).

Tomándose en cuenta los eventos de migración previamente mencionados, existe la probabilidad que la acción del tiempo, combinada a la del ambiente, y de la selección natural y artificial, hayan resultado en un proceso de diferenciación genética frente a las poblaciones criollas ubicadas al norte de Panamá (Criollo de Chiapas, Baja California, Chihuahua, Nayarit, Poblano y Texas Longhorn) y algunas poblaciones de América del Sur (Criollo Casanaré, Ecuatoriano, Criollo Uruguayo, Pampa Chaqueño, Paraguay, Argentino y Patagónico), respecto a las razas panameñas actuales.

El objetivo de este trabajo fue establecer, mediante marcadores microsatélites, la relación genética entre las poblaciones panameñas Guabalá, y Guaymí con algunas poblaciones criollas de Latinoamérica, y 
estimar mediante modelos bayesianos la estructura genética y la proporción de mezcla de algunas poblaciones criollas latinoamericanas y algunas razas cebú.

\section{Materiales y Métodos}

Las poblaciones locales panameñas Guaymí (GY) y Guabalá (GA) fueron descritas previamente por Villalobos Cortés et al. (2009b, 2010, 2011), quienes reportaron por primera vez los valores de diversidad genética intrarracial de ambas razas.

Se utilizó un panel de 27 microsatélites, seleccionados a partir de las recomendaciones hechas por la FAO/ISAG (Food and Agriculture Organization/ International Society of Animal Genetics), para realizar estudios de biodiversidad genética bovina (Food and Agriculture Organization of the United Nations, 2011). Se analizó una base de datos del banco de muestras alélicas de poblaciones bovinas criollas Guaymí, GY (36), y Guabalá, GUA (25), provenientes de Panamá, y se compararon con muestras de las poblaciones criollas al norte de Panamá: Longhorn, LH (42), Criollo Poblano, CPO (43), Criollo de Baja California, CBC (21), Criollo de Nayarit, CNY (24), Criollo de Chiapas, $\mathrm{CHI}$ (30) y muestras de las poblaciones criollas al sur de Panamá: Criollo Casanaré, CC (35), Criollo Ecuatoriano, CE (35), Criollo Uruguayo, CUR (43), Pampa Chaqueño, PA (95), Criollo Paraguay, PGY (36), Criollo Argentino, CAR (41), y Criollo Patagónico, PAT (35). Se incluyeron cinco razas cebuinas, Gyr, GYR (23), Brahman, BRH (36), Sindi, SIN (11), Guzerat, GUZ (15) y Nelore NEL (28), para determinar proporciones de mezcla sobre las poblaciones criollas en uno análisis del programa Structure (Pritchard et al., 2000).

Las muestras fueron obtenidas del laboratorio de genética molecular aplicada del grupo PAI AGR-218, de la Universidad de Córdoba, España. El análisis de la base de datos se realizó en la unidad de bioinformática, del laboratorio de Agrobiotecnología, del Instituto de Investigación Agropecuaria. Se practicó un análisis factorial de correspondencias, mediante el programa Genetix v. 4.02 (Belkhir et al., 2003). Para determinar la estructura genética de las poblaciones, se practicó un análisis de varianza molecular (Amova) y se estimaron los componentes de varianza entre grupos $($ Fst $=\sigma \mathrm{a}+\sigma \mathrm{b} / \sigma \mathrm{t})$, entre poblaciones entre grupos
$(\mathrm{Fsc}=\sigma \mathrm{b} / \sigma \mathrm{b}+\sigma \mathrm{c})$, y total de población entre grupos $\left(\right.$ Fct $\left.=\sigma_{\mathrm{a}} / \sigma_{\mathrm{t}}\right)$, mediante el programa informático Arlequín 3.01 (Excoffier et al., 2005).

También se calcularon el número medio de migrantes por población (Nm) y estadísticos F de Wright (1965) y Weir \& Cockerham (1984) mediante el programa Genetix v. 4.02. Se calcularon las distancias genéticas (Nei et al., 1983) por el programa Populations 1.2.28 (Olivier Langella, (www.cnrsgif.fr/pge/bioinfo/ populations/). Con la matriz obtenida, se crearon árboles de distancias circulares (NeighborNet) con el programa Splits Tree v 4.0 (Bryant \& Moulton, 2004; Huson \& Bryant, 2006).

Se utilizó la versión 2.2.3 del programa Structure (Pritchard et al., 2000), para identificar la estructura de las $\mathrm{K}$ poblaciones utilizadas $\mathrm{y}$, al mismo tiempo, calcular las proporciones de mezcla. Este programa consiste en un modelo de convergencia bayesiano fundamentado en el método de Monte Carlo Cadena de Markov (MCMC), que utiliza modelos basados en agrupamientos de individuos. Se asignó el valor de probabilidad posterior de K (log likelihood; $\operatorname{lnL}$ ) desde 2 hasta 20, con un periodo inicial (burn-in period) de 50 mil iteraciones, sin información previa, y 100 mil iteraciones. Sin embargo, un estudio de Evanno et al. (2005) mostró que la longitud del burn-in y MCMC (cadena de Markov Monte Carlo) de 10 mil cada uno era suficiente para el análisis, y un mayor burn-in o MCMC no cambia significativamente los resultados. Sin embargo, se realizaron tres simulaciones independientes para $\mathrm{K}$ de 2 hasta $20 \mathrm{y}$, con las matrices obtenidas, se obtuvo el alineamiento óptimo de cada una de las tres réplicas mediante el programa Clumpp (Jakobsson \& Rosenberg, 2007).

Se identificó la $\mathrm{K}$ más probable a través de la determinación de la distribución modal de $\Delta K$ (Evanno et al., 2005). También fue estimada la proporción de membresía individual (q) en las poblaciones pre-definidas de cada grupo. Se obtuvo una representación gráfica de los coeficientes de pertenencia individuales, en cada población ancestral con la mayor probabilidad posterior de los datos en cada valor de $\mathrm{K}$.

El análisis también permitió la estimación de individuos puros $(\mathrm{Q} \geq 0,80)$ de los grupos formados, así como el de individuos mezclados $(\mathrm{Q}<0,80)$, entre los diferentes $\mathrm{K}$ (Li et al., 2005). Para la representación 
gráfica de la estructura de la población, se empleó el programa Distruct (Rosenberg, 2004).

\section{Resultados y Discusión}

El eje 1 de análisis factorial de correspondencias explicó el 11,11\% de la varianza total, y el eje 2 el $14,62 \%$, en un total de $25,73 \%$ (Figura 1 ). La variabilidad explicada por los ejes es baja, lo que probablemente es debido a que las poblaciones estudiadas están muy relacionadas (Bos taurus) y provenientes del mismo grupo de animales que llegaron a las islas de las Antillas desde el año 1493. Menores resultados obtuvo Quiroz (2007), al estudiar poblaciones criollas mexicanas: $11,93 \%$ en el eje 1 y $10,94 \%$ en el eje 2 , con un total de $22,87 \%$ de la varianza total. Valores ligeramente más altos reportaron Ginja et al. (2010), al evaluar poblaciones autóctonas portuguesas, con valores de $28,5 \%$ en el eje 1 y $24,2 \%$ en el eje 2 . Sin embargo, en esta investigación, se incluyeron poblaciones de distintos troncos que pudieron incrementar estos valores. Igualmente, $\mathrm{Li}$ et al. (2005), al estudiar cinco razas de la región del norte de Europa y 20 microsatélites, reportaron valores de $43,8 \%$ en el eje 1 y $29,81 \%$ en el eje 2 .

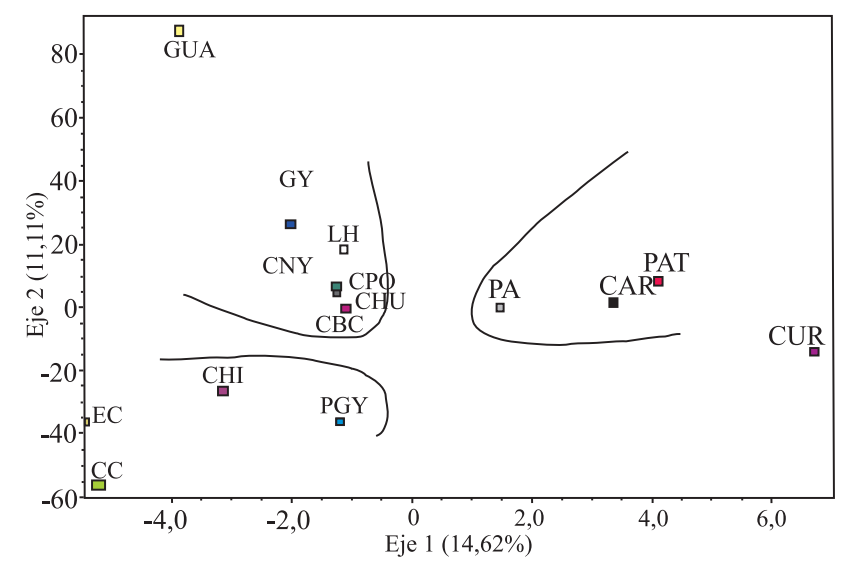

Figura 1. Análisis factorial de correspondencias de poblaciones panameñas y algunas de Latinoamérica de bovinos criollos. GUA, Guabalá ; GY, Guaymí; LH, Texas Longhorn; CPO, Criollo Poblano; CBC, Criollo de Baja California; CHU, Criollo de Chihuahua; CNY, Criollo de Nayarit; CHI, Criollo de Chiapas; CC, Criollo Casanaré; CE, Criollo Ecuatoriano; CUR, Criollo Uruguayo; PA, Pampa Chaqueño; PGY, Criollo Paraguay; CAR, Criollo Argentino; PAT, Criollo Patagónico.
El análisis factorial de correspondencias mostró que las poblaciones Guabalá, y Guaymí se agrupan con los bovinos criollos de la región mesoamericana (razas mexicanas y Texas Longhorn). La diferencia de agrupamiento de las poblaciones mexicanas en comparación con la Criollo de Chiapas fue similar a la reportada por Quiroz (2007). Se puede añadir que el criollo de Chiapas pudo tener un origen geográfico diferente de los demás criollos mexicanos, posiblemente originado de las migraciones de animales desde la región de Panamá, o debido al favorecimiento de algunos alelos en un locus determinado como resultado de la deriva genética (Holsinger \& Weir, 2009). Los grupos formados por Criollo Uruguayo, Pampa Chaqueño, Criollo Argentino y Criollo Patagónico mantienen correspondencia con el área geográfica, salvo el Criollo de Paraguay, que se asoció con los grupos de la región noroeste, Criollo Casanaré y Criollo Ecuatoriano y el de Chiapas, probablemente por efectos ambientales comunes al que se encuentran sometidas las poblaciones, o probablemente por la influencia del cebú (Delgado et al. 2012).

En el Amova, se observó un mayor porcentaje de variación entre grupos de criollas panameñas y las del norte, de 2,63\% ( $<<0,01)$, comparadas con las poblaciones del sur de América con $(1,84 \%, \mathrm{p}<0,01)$. Sin embargo, el porcentaje de variación entre poblaciones dentro de grupos de las Panameñas y las del norte fue de $4,64 \%(p<0,01)$, comparadas con las panameñas y el sur 7,55\% $(\mathrm{p}<0,01)$. Valores más bajos encontró Quiroz (2007) al comparar poblaciones mexicanas y sudamericanas, reportando porcentajes de variación entre grupos de $0,97 \%$. El porcentaje de variación entre poblaciones dentro de grupos fue similar $(7,57 \%)$. Este resultado puede deberse a que las poblaciones de Mesoamérica tuvieron un periodo de ingreso similar hacia tierra firme (año 1521), en el cual predominó la ruta de Panamá y El Río Panuco, proveniente de las Antillas (Quiroz, 2007; Villalobos Cortés et al., 2009b). Es probable la influencia de Pedro Arias de Ávila, primer gobernador de Panamá y posteriormente gobernador de Nicaragua, como promotor de la ganadería y su avance hacia Centroamérica, en los primeros años en que inició la llegada de estos bovinos, y en años subsiguientes hacia América del sur, en la conquista del Perú. Por el contrario, las poblaciones de América del sur presentaron rutas de ingreso tanto en el área del Pacifico como en el Atlántico, aunado a la acción de los 
efectos ambientales y distancias geográficas (Primo, 1992; Delgado et al., 2012). Tomándose en cuenta los resultados previos, se tiene entonces que el índice de fijación $\mathrm{F}_{\mathrm{ST}}$ fue menor entre las poblaciones criollas panameñas y las del norte $(7,26 \%)$ que entre las criollas panameñas y las poblaciones del sur $(9,38 \%)$, valores considerados moderados (Piedrahita et al., 2005). Sin embargo, estos resultados se encuentran dentro del rango correspondiente a los valores de diferenciación genética entre razas. Li et al. (2005), en Noruega, reportaron un índice $\mathrm{F}_{\mathrm{ST}}$ de $6,7 \%$, al estudiar razas autóctonas del Norte de Europa; Ulloa-Arvizu et al. (2008) reportaron índice de $\mathrm{F}_{\mathrm{ST}}$ de $8 \%$, al comparar algunas razas criollas mexicanas con algunas poblaciones de la Península Ibérica. Los valores mas bajos de $\mathrm{F}_{\mathrm{ST}}$ obtenidos entre las razas panameñas y de norte se pueden explicar por los valores encontrados entre la raza Guaymí y Longhorn $(0,044)$, Criollo Poblano $(0,042)$, Criollo Baja California $(0,021)$, Criollo Chihuahua $(0,024)$ y Criollo de Nayarit $(0,044)$, cuyos valores fueron menores que los observados entre Criollo Guaymí y Guabalá $(0,056)$, conforme Quadro 1.

En general, se observa que la raza Guabalá presenta mayores valores de diferenciación $\mathrm{F}_{\mathrm{ST}}$, probablemente por un aislamiento reproductivo mucho más estricto que el observado en el Guaymí. Por muchos años, la raza Guabalá estuvo en manos de una sola familia, que mantuvo mayores controles que sobre la Guaymí, que vivía de manera extensiva en las regiones montañosas de la comarca Gnöbe-Bugle.

Los valores de $\mathrm{Nm}$ complementan los resultados observados en $\mathrm{F}_{\text {ST }} \mathrm{y}$ mostran mayor flujo de genes entre las poblaciones del norte con las poblaciones panameñas. La correlación negativa entre los valores $\mathrm{F}_{\text {ST }}$ y $\mathrm{Nm}$ permiten establecer de manera más precisa las relaciones entre poblaciones, particularmente en aquellas estrechamente relacionadas en términos evolutivos y donde la deriva genética ha sido un factor importante (Mukesh et al., 2004). Utilizándose ambos indicadores, se establece que con menores valores de $\mathrm{F}_{\text {Sт }}$ y mayores valores de $\mathrm{Nm}$ las poblaciones son mas relacionadas y viceversa (Fraga Nodarse et al., 2009). Los valores más altos de $\mathrm{Nm}$ se encontraron entre Guaymí con el Criollo Poblano $(6,01)$, y para Guabalá con Criollo Casanaré $(2,43)$, conforme Quadro 1 encima de la diagonal. Similares resultados obtuvieron Mukesh et al. (2004), al estudiar poblaciones cebuinas en India, quienes reportaron valores de diferenciación genética de 0,053 a 0,065 apoyados con valores de Nm de 2,71 a 4,48; igualmente, Xhang et al. 2007 estudiaron poblaciones locales en China y encontraron valores de $F_{\text {ST }}$ que oscilaron entre 0,01 a 0,24 , aunque en los valores de Nm se observaron más altos $(0,82-3,14)$.

Los resultados del análisis de distancia genética $\mathrm{D}_{\mathrm{A}}$ de Nei (Quadro 2) son similares a los obtenidos en el Amova y en el análisis factorial de correspondencia,

Quadro 1. Valores de Nm (Wright, 1965), encima de la diagonal, y de $\mathrm{F}_{\mathrm{ST}}$, debajo de la diagonal, de poblaciones panameñas y latinoamericanas de bovinos criollos.

\begin{tabular}{lccccccccccccccc}
\hline & GUA & GY & LH & CPO & CBC & CHU & CNY & CHI & CC & CE & CUR & PA & PGY & CAR & PAT \\
\hline GUA & - & 3,40 & 2,23 & 2,07 & 1,76 & 1,99 & 2,38 & 2,31 & 2,43 & 1,80 & 1,20 & 2,24 & 1,94 & 1,37 & 1,38 \\
GY & 0,056 & - & 5,10 & 6,01 & 3,67 & 4,97 & 5,52 & 4,01 & 5,75 & 3,76 & 2,23 & 4,99 & 5,25 & 2,49 & 2,41 \\
LH & 0,089 & 0,044 & - & 6,21 & 5,59 & 6,83 & 6,48 & 3,65 & 4,00 & 2,42 & 2,09 & 4,60 & 3,84 & 2,77 & 2,09 \\
CPO & 0,113 & 0,047 & 0,042 & - & 9,04 & 12,37 & 7,95 & 5,40 & 5,64 & 3,30 & 2,49 & 5,40 & 4,91 & 2,95 & 2,44 \\
CBC & 0,093 & 0,021 & 0,024 & 0,028 & - & 11,90 & 6,99 & 4,60 & 4,64 & 3,06 & 2,19 & 4,77 & 4,32 & 2,74 & 2,08 \\
CHU & 0,091 & 0,024 & 0,024 & 0,028 & 0,026 & - & 13,07 & 6,19 & 5,22 & 3,51 & 2,37 & 5,84 & 5,19 & 3,00 & 2,10 \\
CNY & 0,084 & 0,039 & 0,037 & 0,041 & 0,025 & 0,010 & - & 6,46 & 5,87 & 3,54 & 2,29 & 5,06 & 5,65 & 2,70 & 2,15 \\
CHI & 0,099 & 0,076 & 0,073 & 0,070 & 0,067 & 0,038 & 0,037 & - & 4,61 & 4,95 & 2,43 & 4,87 & 5,60 & 2,51 & 1,95 \\
CC & 0,108 & 0,041 & 0,061 & 0,043 & 0,026 & 0,032 & 0,045 & 0,060 & - & 3,74 & 1,88 & 4,40 & 5,92 & 2,42 & 2,33 \\
CE & 0,131 & 0,069 & 0,099 & 0,091 & 0,067 & 0,055 & 0,060 & 0,054 & 0,068 & - & 1,88 & 3,11 & 6,90 & 1,71 & 1,39 \\
CUR & 0,177 & 0,111 & 0,128 & 0,121 & 0,114 & 0,103 & 0,109 & 0,109 & 0,127 & 0,113 & - & 3,22 & 3,10 & 2,20 & 1,85 \\
PA & 0,107 & 0,057 & 0,061 & 0,058 & 0,024 & 0,030 & 0,045 & 0,055 & 0,052 & 0,080 & 0,084 & - & 4,82 & 3,31 & 2,44 \\
PGY & 0,132 & 0,058 & 0,070 & 0,070 & 0,050 & 0,036 & 0,045 & 0,054 & 0,049 & 0,028 & 0,069 & 0,058 & - & 3,57 & 2,60 \\
CAR & 0,154 & 0,093 & 0,042 & 0,095 & 0,071 & 0,070 & 0,083 & 0,090 & 0,093 & 0,117 & 0,103 & 0,054 & 0,064 & - & 1,93 \\
PAT & 0,163 & 0,109 & 0,024 & 0,097 & 0,069 & 0,069 & 0,101 & 0,119 & 0,109 & 0,134 & 0,087 & 0,083 & 0,088 & 0,093 & - \\
\hline
\end{tabular}

GUA, Guabalá; GY, Guaymí; LH, Texas Longhorn; CPO, Criollo Poblano; CBC, Criollo de Baja California; CHU, Criollo de Chihuahua; CNY, Criollo de Nayarit; CHI, Criollo de Chiapas; CC, Criollo Casanaré; CE, Criollo Ecuatoriano; CUR, Criollo Uruguayo; PA, Pampa Chaqueño; PGY, Criollo Paraguay; CAR, Criollo Argentino; PAT, Criollo Patagónico, 
observándose una menor distancia entre las poblaciones del norte y las panameñas, en comparación con las poblaciones del sur, particularmente en las poblaciones Guaymí con Longhorn $(0,14)$, y en Criollo Poblano $(0,13)$ y Criollo Nayarit $(0,141)$. Cabe destacar que las poblaciones de Criollo Patagónico y Criollo Uruguayo tuvieron mayor distancia genética con el Criollo Guaymí (0,216 y 0,205 respectivamente) y Criollo Uruguayo y Criollo Ecuatoriano con el Guabalá (0,307 y 0,281 respectivamente), lo que coincidió con la mayor distancia geográfica. Aunque las poblaciones Guaymí y Guabalá estén localizadas dentro de la misma región, se observaron menores valores de distancia de otras poblaciones que entre ellas, confirmando sus diferencias genéticas y las presiones ambientales a que fueron sometidas. El efecto de aislamiento geográfico y reproductivo mostró una diferencia entre ambas poblaciones, sumando probablemente la deriva genética y cruzamientos aleatorios en las primeras etapas de la formación de estas razas, lo que marcó la diferenciación entre ambas, años después.

La Figura 2, del árbol de distancias circulares muestra cuatro agrupamientos: 1, Guaymí y Guabalá; 2, criollas del Cono Sur (Criollo Uruguayo, Criollo Argentino, Criollo Patagónico y Pampa Chaqueño); 3, las criollas Mexicanas y el Texas Longhorn; 4, Criollo de Paraguay, Casanaré, Ecuatoriano y el Criollo de Chiapas. La conformación del árbol de distancias circulares fue similar a lo observado en el análisis factorial de correspondencia, donde también se presenta el agrupamiento del Criollo de Chiapas y las poblaciones de Suramérica de la zona del Pacífico (Casanaré y Ecuatoriano) y el Criollo de Paraguay. También se observa el agrupamiento correspondiente al área geográfica entre las poblaciones Guaymí y Guabalá en Panamá, y las poblaciones mexicanas y el Texas Longhorn (excepto el Criollo de Chiapas). Ambos grupos corresponden a los primeros ingresos de bovinos a tierra firme, en el año 1521, por el Río Panuco - desde la Isla de la Fernandina (Cuba), llevado a cabo por Gregorio de Villalobos -, y por el lado del Pacífico - en la región de Nayarit en 1565 - hacia Baja California, en 1670 (Quiroz, 2007; Ulloa-Arvizu et al., 2008), y desde Isla de Santiago (Jamaica) hacia Santa María la Antigua del Darién, en Panamá, solicitado por Pedro Arias de Ávila (Villalobos Cortés et al., 2009a).

La Figura 3 muestra las proporciones de mezcla para $\mathrm{K}=2, \mathrm{~K}=5 \mathrm{y} \mathrm{K}=17$. Cada animal se encuentra representado por líneas verticales, divididas en $\mathrm{K}$ colores, donde $\mathrm{K}$ es el número de grupos asumidos y los colores muestran de la proporción de cada individuo perteneciente a un grupo. Cuando el valor fue de $K=2$, se separaron dos grupos correspondientes a poblaciones Bos indicus (Gyr, Nelore, Brahman, Guzerat y Sindi) y poblaciones Bos taurus (Guabalá, Guaymí, T. Longhorn, Criollo Poblano, Criollo Baja California, Criollo de Chihuahua, Criollo de Nayarit, Criollo Uruguayo, Pampa Chaqueño, Criollo Argentino y Criollo Patagónico). Cuando $\mathrm{K}=5$, las poblaciones panameñas Guaymí y Guabalá formaron un grupo, y

Quadro 2. Distancias genéticas (Nei et al., 1983) de poblaciones panameñas y latinoamericanas de bovinos criollos.

\begin{tabular}{|c|c|c|c|c|c|c|c|c|c|c|c|c|c|c|}
\hline & GY & LH & $\mathrm{CPO}$ & $\mathrm{CBC}$ & $\mathrm{CHU}$ & $\mathrm{CNY}$ & $\mathrm{CHI}$ & $\mathrm{CC}$ & $\mathrm{CE}$ & CUR & $\mathrm{PA}$ & PGY & CAR & PAT \\
\hline$\overline{\text { GUA }}$ & 0,153 & 0,223 & 0,219 & 0,248 & 0,243 & 0,223 & 0,234 & 0,244 & 0,281 & 0,307 & 0,210 & 0,252 & 0,260 & 0,272 \\
\hline GY & - & 0,140 & 0,130 & 0,163 & 0,155 & 0,141 & 0,169 & 0,156 & 0,192 & 0,205 & 0,126 & 0,147 & 0,187 & 0,216 \\
\hline LH & & - & 0,108 & 0,118 & 0,121 & 0,127 & 0,175 & 0,163 & 0,260 & 0,211 & 0,134 & 0,176 & 0,152 & 0,197 \\
\hline $\mathrm{CPO}$ & & & - & 0,110 & 0,100 & 0,105 & 0,132 & 0,143 & 0,220 & 0,201 & 0,110 & 0,146 & 0,156 & 0,187 \\
\hline $\mathrm{CBC}$ & & & & - & 0,121 & 0,123 & 0,148 & 0,166 & 0,226 & 0,215 & 0,129 & 0,164 & 0,181 & 0,214 \\
\hline $\mathrm{CHU}$ & & & & & - & 0,112 & 0,163 & 0,175 & 0,243 & 0,228 & 0,132 & 0,166 & 0,177 & 0,220 \\
\hline $\mathrm{CNY}$ & & & & & & - & 0,135 & 0,146 & 0,230 & 0,219 & 0,130 & 0,137 & 0,185 & 0,215 \\
\hline $\mathrm{CHI}$ & & & & & & & - & 0,148 & 0,190 & 0,236 & 0,134 & 0,146 & 0,198 & 0,245 \\
\hline $\mathrm{CC}$ & & & & & & & & - & 0,212 & 0,258 & 0,142 & 0,126 & 0,196 & 0,206 \\
\hline $\mathrm{CE}$ & & & & & & & & & - & 0,316 & 0,199 & 0,172 & 0,291 & 0,337 \\
\hline CUR & & & & & & & & & & - & 0,161 & 0,197 & 0,166 & 0,180 \\
\hline PA & & & & & & & & & & & - & 0,121 & 0,136 & 0,175 \\
\hline PGY & & & & & & & & & & & & - & 0,157 & 0,196 \\
\hline CAR & & & & & & & & & & & & & - & 0,167 \\
\hline
\end{tabular}

GUA, Guabalá ; GY, Guaymí; LH, Texas Longhorn; CPO, Criollo Poblano; CBC, Criollo de Baja California; CHU, Criollo de Chihuahua; CNY, Criollo de Nayarit; CHI, Criollo de Chiapas; CC, Criollo Casanaré; CE, Criollo Ecuatoriano; CUR, Criollo Uruguayo; PA, Pampa Chaqueño; PGY, Criollo Paraguay; CAR, Criollo Argentino; PAT, Criollo Patagónico. 
el Criollo Argentino y Criollo Patagónico formaron otro grupo junto con el Pampa Chaqueño. El Criollo Ecuatoriano se mantuvo agrupado con las razas cebú. Cuando $\mathrm{K}=17$, todas las poblaciones se separaron, excepto Criollo de Chihuahua con el Criollo de Nayarit,

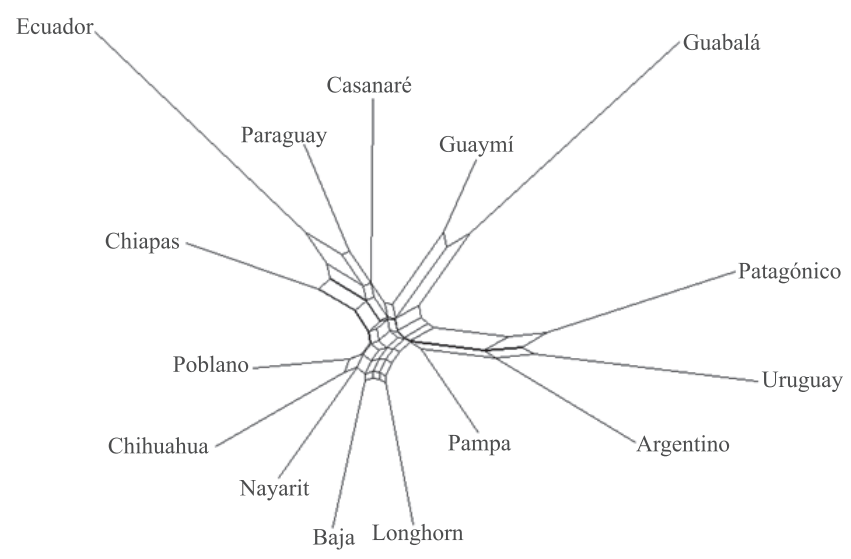

Figura 2. Splitgraph con el método NeighborNet de poblaciones bovinas criollas panameñas, del norte y del sur de América. y el criollo ecuatoriano mostró que parte de su genoma es compartido con el de los grupos cebú.

La representación gráfica mostró comportamiento diferente a lo mencionado por Quiroz (2007), cuando comparó poblaciones mexicanas y 17 razas de Iberoamérica y reportó que las poblaciones mexicanas no se separaron, sino que se mantuvieron en un solo grupo, probablemente por no haber alcanzado la máxima $\mathrm{K}$ en el análisis utilizado. Sin embargo, Martínez (2008) reportó un comportamiento similar en las razas Criollo Patagónico y Criollo Argentino y mostró una separación en un valor de $K$ menor $(K=10)$, pero la cantidad de razas incluidas en el estudio fue menor $(n=10)$. Los resultados basados en el cálculo de Delta $\mathrm{K}$ con el método de Evanno et al. (2005) indican que para las 20 razas estudiadas el número más probable de poblaciones es de 17 y que la subdivisión más probable fue debido a las razas o grupos más relacionados genéticamente. Todos los grupos se separaron, a excepción del Criollo de Chihuahua con el Criollo de Nayarit, que resultaron estar muy relacionados, como se observa en los valores de $\mathrm{F}_{\mathrm{ST}}(0,01)$, o probablemente unidos muy cercanamente a un ancestro común. El
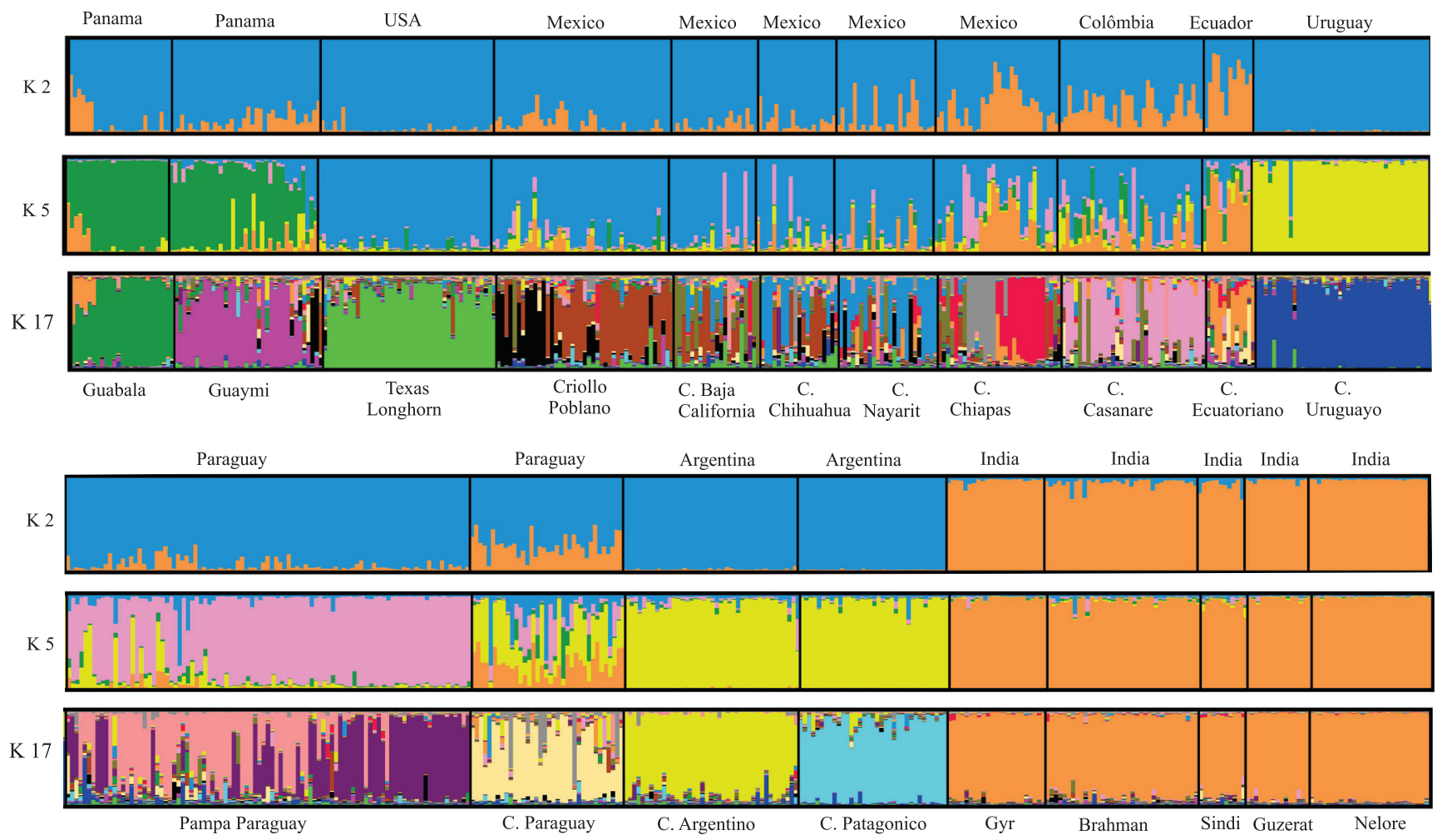

Figura 3. Estructura de 20 poblaciones criollas y cebuinas, mediante el programa Structure, basado en modelos bayesianos (Pritchard et al., 2000) y graficado mediante Distruct (Rosenberg, 2004). 
criollo Ecuatoriano se mantuvo relacionado con las poblaciones cebú en todos los cálculos de K.

Los resultados del análisis bayesiano (Quadro 3) muestran que los valores medios de $\mathrm{Q}$, en cada uno de los 17 grupos mostrados para las diferentes razas, oscilaron desde 0,29, en el Criollo de Baja California, hasta 0,88 , en el Criollo Uruguayo, en el grupo de poblaciones Bos taurus, y desde 0,84 hasta 0,95 para el Sindi y Nelore, respectivamente, en el grupo de las poblaciones Bos indicus.

Los valores más altos de Q fueron observados cuando $\mathrm{K}=17$, en el Criollo Uruguayo $(0,88)$, Criollo Patagónico $(0,86)$, Criollo Argentino $(0,84)$, Criollo Guabalá $(0,82)$ y Texas Longhorn $(0,78)$. El resto de las poblaciones mostraron valores de $\mathrm{Q}$ considerados bajos.

Los resultados obtenidos en los criollos argentinos, mediante el modelo aplicado, fueron menores con lo reportado por Martínez (2008), en Argentina, con valores de Q de 0,92 y 0,91 para el Criollo Patagónico y Argentino, respectivamente. No fue posible observar diferencias entre el Criollo Chihuahua y el Criollo de Nayarit, los cuales se mantuvieron en el mismo grupo y mostraron un alto nivel de mezcla $(Q=0,31$ y 0,45 , respectivamente). Igualmente, las demás poblaciones mexicanas (Criollo Poblano, Criollo Baja California y Criollo de Chiapas) mostraron altos niveles de mezcla. Quiroz (2007) reportó valores altos de mezcla en las poblaciones mexicanas, particularmente entre el Criollo de Nayarit, Chihuahua y Baja California. Similares resultados reportó Delgado et al. (2012) sobre las razas mexicanas Nayarit, Chihuahua y Baja California, que no pudieron ser diferenciadas en el análisis, y la mezcla de cebú sobre las poblaciones criollas de Chiapas y Ecuador, probablemente por la gran influencia ejercida durante los inicios del siglo XX, cuando las poblaciones cebuinas fueron consideradas razas adaptadas a ambientes tropicales y por el efecto de heterosis.

En el caso del Criollo Guaymí, niveles bajos de Q fueron observados, pero mayores que los observados en las poblaciones mexicanas. Se requiere el desarrollo de nuevas estrategias de conservación y manejo de los hatos, para evitar la pérdida de diversidad y el cruzamiento indeseado con otras razas, como se pudo observar, previamente a los trabajos de Villalobos Cortés et al. (2010), cuando se tomaban ambas razas como una sola, y ocurrían cruzamientos de algunos sementales Guabalá con hembras Guaymí, situación que fue rápidamente corregida. La raza Guabalá presenta una mejor

Quadro 3. Estimación de las proporción de miembros de cada grupo (Q), según se infiere mediante el programa Structure (Pritchard et al., 2000), para $\mathrm{K}=17$.

\begin{tabular}{|c|c|c|c|c|c|c|c|c|c|c|c|c|c|c|c|c|c|}
\hline \multirow[t]{2}{*}{$\overline{\text { Raza }}$} & \multicolumn{17}{|c|}{ Grupos inferidos $^{(1)}$} \\
\hline & 1 & 2 & 3 & 4 & 5 & 6 & 7 & 8 & 9 & 10 & 11 & 12 & 13 & 14 & 15 & 16 & 17 \\
\hline & \multicolumn{17}{|c|}{ Bos taurus } \\
\hline Guabalá & 0,00 & 0,01 & 0,02 & 0,01 & 0,01 & 0,01 & 0,00 & $\underline{0,82}$ & 0,01 & 0,01 & 0,01 & 0,08 & 0,01 & 0,01 & 0,01 & 0,01 & 0,01 \\
\hline Guaymí & 0,02 & 0,01 & $\underline{0,62}$ & 0,02 & 0,06 & 0,03 & 0,04 & 0,05 & 0,01 & 0,02 & 0,02 & 0,02 & 0,01 & 0,02 & 0,02 & 0,01 & 0,01 \\
\hline Texas Longhorn & $\underline{0,78}$ & 0,01 & 0,02 & 0,01 & 0,02 & 0,01 & 0,04 & 0,01 & 0,01 & 0,02 & 0,01 & 0,00 & 0,01 & 0,02 & 0,02 & 0,01 & 0,01 \\
\hline Criollo Poblano & 0,04 & 0,01 & 0,02 & 0,02 & 0,26 & 0,02 & $\underline{0,45}$ & 0,01 & 0,01 & 0,04 & 0,02 & 0,01 & 0,02 & 0,02 & 0,01 & 0,01 & 0,02 \\
\hline Criollo Baja California & 0,08 & 0,01 & 0,02 & 0,01 & 0,05 & 0,02 & 0,20 & 0,01 & 0,03 & $\underline{0,29}$ & 0,01 & 0,01 & 0,04 & 0,12 & 0,02 & 0,07 & 0,03 \\
\hline Criollo Chihuahua & 0,06 & 0,02 & 0,02 & 0,02 & 0,09 & 0,02 & 0,20 & 0,01 & 0,04 & 0,03 & 0,03 & 0,03 & 0,02 & $\underline{0,31}$ & 0,02 & 0,03 & 0,06 \\
\hline Criollo Nayarit & 0,04 & 0,01 & 0,04 & 0,01 & 0,10 & 0,03 & 0,05 & 0,01 & 0,01 & 0,07 & 0,01 & 0,06 & 0,05 & $\underline{0,45}$ & 0,01 & 0,04 & 0,03 \\
\hline Criollo Chiapas & 0,02 & 0,01 & 0,01 & 0,01 & 0,02 & 0,01 & 0,06 & 0,01 & 0,02 & 0,10 & 0,01 & 0,05 & $\underline{0,39}$ & 0,03 & 0,01 & 0,02 & 0,23 \\
\hline Criollo Casanaré & 0,02 & 0,01 & 0,02 & 0,01 & 0,02 & 0,04 & 0,02 & 0,01 & 0,01 & 0,10 & $\underline{0,59}$ & 0,03 & 0,02 & 0,02 & 0,02 & 0,05 & 0,03 \\
\hline Criollo Ecuatoriano & 0,02 & 0,02 & 0,06 & 0,01 & 0,02 & 0,16 & 0,04 & 0,04 & 0,03 & 0,05 & 0,03 & $\underline{0,40}$ & 0,06 & 0,02 & 0,01 & 0,03 & 0,03 \\
\hline Criollo Uruguayo & 0,02 & $\underline{0,88}$ & 0,01 & 0,01 & 0,01 & 0,01 & 0,01 & 0,00 & 0,01 & 0,01 & 0,00 & 0,00 & 0,00 & 0,01 & 0,02 & 0,01 & 0,01 \\
\hline Pampa Chaqueño & 0,01 & 0,03 & 0,01 & 0,02 & 0,02 & 0,03 & 0,02 & 0,02 & $\underline{0,41}$ & 0,02 & 0,01 & 0,01 & 0,01 & 0,01 & 0,02 & 0,35 & 0,02 \\
\hline Criollo Paraguay & 0,01 & 0,01 & 0,01 & 0,02 & 0,01 & $\underline{0,63}$ & 0,01 & 0,01 & 0,01 & 0,01 & 0,03 & 0,04 & 0,01 & 0,02 & 0,05 & 0,02 & 0,10 \\
\hline Criollo Argentino & 0,01 & 0,02 & 0,01 & 0,02 & 0,01 & 0,01 & 0,01 & 0,01 & 0,02 & 0,01 & 0,01 & 0,00 & 0,01 & 0,01 & $\underline{0,84}$ & 0,01 & 0,01 \\
\hline \multirow[t]{2}{*}{ Criollo Patagónico } & 0,01 & 0,02 & 0,01 & $\underline{0,86}$ & 0,01 & 0,01 & 0,01 & 0,01 & 0,01 & 0,01 & 0,01 & 0,00 & 0,00 & 0,01 & 0,03 & 0,01 & 0,01 \\
\hline & \multicolumn{17}{|c|}{ Bos indicus } \\
\hline Gyr & 0,01 & 0,00 & 0,01 & 0,00 & 0,01 & 0,01 & 0,01 & 0,00 & 0,01 & 0,01 & 0,01 & $\underline{0,91}$ & 0,01 & 0,01 & 0,00 & 0,01 & 0,01 \\
\hline Brahman & 0,01 & 0,01 & 0,01 & 0,00 & 0,01 & 0,01 & 0,01 & 0,00 & 0,01 & 0,01 & 0,01 & $\underline{0,87}$ & 0,01 & 0,01 & 0,00 & 0,01 & 0,01 \\
\hline Sindi & 0,01 & 0,01 & 0,01 & 0,01 & 0,01 & 0,03 & 0,01 & 0,01 & 0,01 & 0,01 & 0,02 & $\underline{0,84}$ & 0,02 & 0,01 & 0,00 & 0,01 & 0,01 \\
\hline Guzerat & 0,00 & 0,00 & 0,01 & 0,00 & 0,01 & 0,01 & 0,00 & 0,00 & 0,01 & 0,00 & 0,02 & $\underline{0,92}$ & 0,00 & 0,00 & 0,00 & 0,00 & 0,00 \\
\hline Nelore & 0,00 & 0,00 & 0,00 & 0,00 & 0,00 & 0,01 & 0,00 & 0,00 & 0,00 & 0,00 & 0,00 & $\underline{0,95}$ & 0,00 & 0,00 & 0,00 & 0,00 & 0,00 \\
\hline
\end{tabular}

${ }^{(1)}$ La contribución del grupo más importante por raza está representada en subrayado. 
perspectiva hacia su futuro, debido a que es manejada por productores privados, y se ha mostrado un gran interés por su conservación y fomento. Cabe destacar que esta raza Guabalá se encuentra dentro del grupo de razas con los niveles de Q considerados como puras.

\section{Conclusiones}

1. Hay una estrecha relación histórica, geográfica y genética entre las poblaciones panameñas, criollas mexicanas y Texas Longhorn, a partir de las migraciones de sus precursores desde las Antillas hacia Panamá y México.

2. Las poblaciones con mayores valores de pureza son el Criollo Uruguayo, Criollo Patagónico, Criollo Argentino, Criollo Guabalá y Texas Longhorn.

3. El Criollo Ecuatoriano y de Chiapas muestran que una proporción de su genoma tenía influencia de las razas cebú.

4. En general, los agrupamientos observados muestran una gran concordancia con el área geográfica de las razas estudiadas.

\section{Agradecimientos}

A la Universidad de Córdoba, la Red Conbiand, al Instituto de Investigación Agropecuaria de Panamá y la Secretaria Nacional de Ciencia y Tecnología de Panamá, por el financiamiento y la colaboración en la realización de este trabajo.

\section{Referencias}

ARCHIVO GENERAL DE INDIAS. Envío de ganados de Jamaica a Panamá. Disponible en: <http://pares.mcu.es/ ParesBusquedas/servlets/Control_servlet?accion=3\&txt_id_ desc_ud $=376072 \&$ fromagenda $=\mathrm{N}>$. Accedido el: 20 agosto 2007.

BELKHIR, K.; BORSA, P.; CHIKHI, P.; RAUFASTE, N.; BONHOMME, F. Genetix: logiciel sous Windows pour la génétique des populations. Version 4.05. Montpellier: Université de Montpellier, 2003.

BRYANT, D.; MOULTON, V. Neighbor-Net: an agglomerative method for the construction of phylogenetic networks. Molecular Biology and Evolution, v.21, p.255-265, 2004.

CORTÉS RAMOS, A. Sociedad y naturaleza antes y después de la conquista y colonización de América Central. 2002. Disponible en: <http://www.ciicla.ucr.ac.cr/recursos/docs/ biblioteca/revista-intercambio/anol/presentacion.pdf $>$. Accedido el: 25 sept. 2012.

DELGADO, J.V.; MARTÍNEZ, A.M.; ACOSTA, A.; ALVAREZ, L.A.; ARMSTRONG, E.; CAMACHO, E.; CAÑÓN,
J.; CORTÉS, O.; DUNNER, S.; LANDI, V.; MARQUES, J.R.; MARTÍN-BURRIEL, I.; MARTÍNEZ, O.R.; MARTÍNEZ, R.D.; MELUCCI,L.;MUNOZZ,J.E.;PENEDO,M.C.T.;POSTIGLIONI, A.; QUIRÓZ, J.; RODELLAR, C.; SPONENBERG, P.; UFFO, O.; ULLOA-ARVIZU, R.; VEGA-PLA, J.L.; VILLALOBOS, A.; ZAMBRANO, D.; ZARAGOZA, P.; GAMA, L.T.; GINJA, C. Genetic characterization of Latin-American Creole cattle using microsatellite markers. Animal Genetics, v.43, p.2-10, 2012.

EVANNO, G.; REGNAUT, S.; GOUDET, J. Detecting the number of clusters of individuals using the software STRUCTURE: a simulation study. Molecular Ecology, v.14, p.2611-2620, 2005.

EXCOFFIER, L.; LAVAL, G.; SCHNEIDER, S. Arlequin (version 3.0): an integrated software package for population genetics data analysis. Evolutionary Bioinformatics, v.1, p.47-50, 2005.

FALUSH, D.; STEPHENS, M.; PRITCHARD, J.K. Inference of population structure using multilocus genotype data: linked loci and correlated allele frequencies. Genetics, v.164, p.1567-1587, 2003.

FOOD AND AGRICULTURE ORGANIZATION OF THE UNITED NATIONS. Molecular genetic characterization of animal genetic resources. Roma: FAO, 2011. 68p. (FAO. Animal production and health guidelines, 9).

FRAGA NODARSE, J.; RODRÍGUEZ RODRIGUEZ, J.; FUENTES GONZÁLEZ, O.; CASTEX RODRIGUEZ, M.; FERNÁNDEZ-CALIENES VALDÉS, A. Variabilidad genética de poblaciones de Triatoma flavida (Hemiptera: Reduviidae) en la península de Guanahacabibes. Revista Cubana de Medicina Tropical, v.61, p.88-96, 2009.

GINJA, C.; PENEDO, M.C.T.; SOBRAL, M.F.; MATOS, J.; BORGES, C.; NEVES, D.; RANGEL-FIGUEIREDO, T.; CRAVADOR, A. Molecular genetic analysis of a cattle population to reconstitute the extinct Algarvia breed. Genetics Selection Evolution, v.42, 2010. Doi: 10.1186/1297-9686-42-18.

GUILHERMO, C.A. Panamá: agua y desarrollo en vísperas del segundo siglo. Publicación Seriada Tareas, n.114, p.21-52, 2003.

HOLSINGER, K.E.; WEIR, B.S. Genetics in geographically structured populations: defining, estimating and interpreting $\mathrm{F}_{\mathrm{ST}}$ . Nature Reviews Genetics, v.6, p.639-650, 2009.

HUSON, D.H.; BRYANT, D. Application of phylogenetic networks in evolutionary studies. Molecular Biology and Evolution, v.23, p.254-267, 2006.

JAKOBSSON, M.; ROSENBERG, N.A. CLUMPP: a cluster matching and permutation program for dealing with label switching and multimodality in analysis of population structure. Bioinformatics, v.23, p.1801-1806, 2007.

LI, M.H.; STERNBAUER, K.; HAAHR, P.T.; KANTANEN, J. Genetic components in contemporary Faroe Islands Cattle as revealed by microsatellite analysis. Journal of Animal and Breeding Genetics, v.122, p.309-317, 2005. 
MARTÍNEZ, R.D. Caracterización genética y morfológica del bovino criollo argentino de origen patagónico. 2008. 244p. Tesis (Doctoral) - Universidad Politécnica de Valencia, Valencia.

MUKESH, M.; SODHI, M.; BHATIA, S.; MISHRA, B.P. Genetic diversity of Indian native cattle breeds as analysed with 20 microsatellite loci. Journal of Animal Breeding and Genetics, v.121, p.416-424, 2004.

NEI, M.; TAJIMA, F.; TATEN, Y. Accuracy of estimated phylogenetic trees from molecular data. Journal of Molecular Evolution, v.19, p.153-170, 1983.

OLSON, T.A.; LUCENA, C.; CHASE JUNIOR, C.C.;HAMMOND, A.C. Evidence of a major gene influencing hair length and heat tolerance in Bos taurus cattle. Journal of Animal Science, v.81, p.80-90, 2003.

PIEDRAHITA, A.M.; MUÑOZ, J.E.; ALVAREZ, A.; POSSO, A.M. Caracterización molecular de ganado Hartón del Valle usando marcadores moleculares RAMS. Facultad de Ciencias Agropecuarias, v.3, p.19-31, 2005.

PRIMO, A.T. El ganado bovino Iberico en las Americas: 500 años después. Archivos de Zootecnia, v.41, p.421-432, 1992.

PRITCHARD, J.K.; STEPHENS, M.; DONNELLY, P. Inference of population structure using multilocus genotype data. Genetics, v.155, p.945-959, 2000.

QUIROZ, V.J. Caracterización genética de los bovinos Criollos mexicanos y su relación con otras poblaciones bovinas. 2007. 155p. Tesis (Doctoral) - Universidad de Córdoba, Córdoba.

RODERO, A.; DELGADO, J.V.; RODERO, E. Primitive andalusian livestock and their implications in the discovery of America. Archivos de Zootecnia, v.41, p.383-400, 1992.
ROSENBERG, N. DISTRUCT: a program for the graphical display of population Structure. Molecular Ecology Notes, v.4, p.137-138, 2004.

ULLOA-ARVIZU, R.; GAYOSSO-VAZQUEZ, A.; RAMOSKURI, M.; ESTRADA, F.J.; MONTANO, M.; ALONSO, R.A. Genetic analysis of Mexican Criollo cattle populations. Journal of Animal Breeding and Genetics, v.125, p.351-359, 2008.

VILLALOBOS CORTÉS, A.I. Caracterización genética de las poblaciones bovinas Guaymí y Guabalá y su relación con otras poblaciones bovinas mediante microsatélites. 2010. 173p. Tesis (Doctoral) - Universidad de Córdoba, Córdoba.

VILLALOBOS CORTÉS, A.I.; MARTÍNEZ, A.M.; DELGADO, J.V. Historia de los bovinos en Panamá y su relación con las poblaciones bovinas de Iberoamérica. Archivos de Zootecnia, v.58, p.121-129, 2009a.

VILLALOBOS CORTÉS, A.I.; MARTÍNEZ, A.M.; ESCOBAR, C.; VEGA-PLA, J.L.; DELGADO, J.V. Study of genetic diversity of the Guaymi and Guabala bovine populations by means of microsatellites. Livestock Science, v.131, p.45-51, 2010.

VILLALOBOS CORTÉS, A.I.; MARTÍNEZ, A.M.; VEGA-PLA, J.L.; DELGADO, J.V. Genetic characterization of the Guabala bovine population with microsatellites. Archivos de Zootecnia, v.58, p.485-488, 2009b.

VILLALOBOS CORTÉS, A.I.; MARTÍNEZ A.M.; VEGA-PLA, J.L.; DELGADO, J.V. Genetic structure and bottleneck of the Guaymi bovine population by means of microsatellites. Archivos de Zootecnia, v.60, p.767-775, 2011.

WEIR, B.S.; COCKERHAM, C.C. Estimating F-statistics for the analysis of population structure. Evolution, v.38, p.1358-1370, 1984.

WRIGHT, S. The interpretation of population structure by F-statistics with special regard to systems of mating. Evolution, v. 19, p.395-420,1965.

Recibido en 25 de marzo de 2011 y aprobado en 18 de septiembre de 2012 